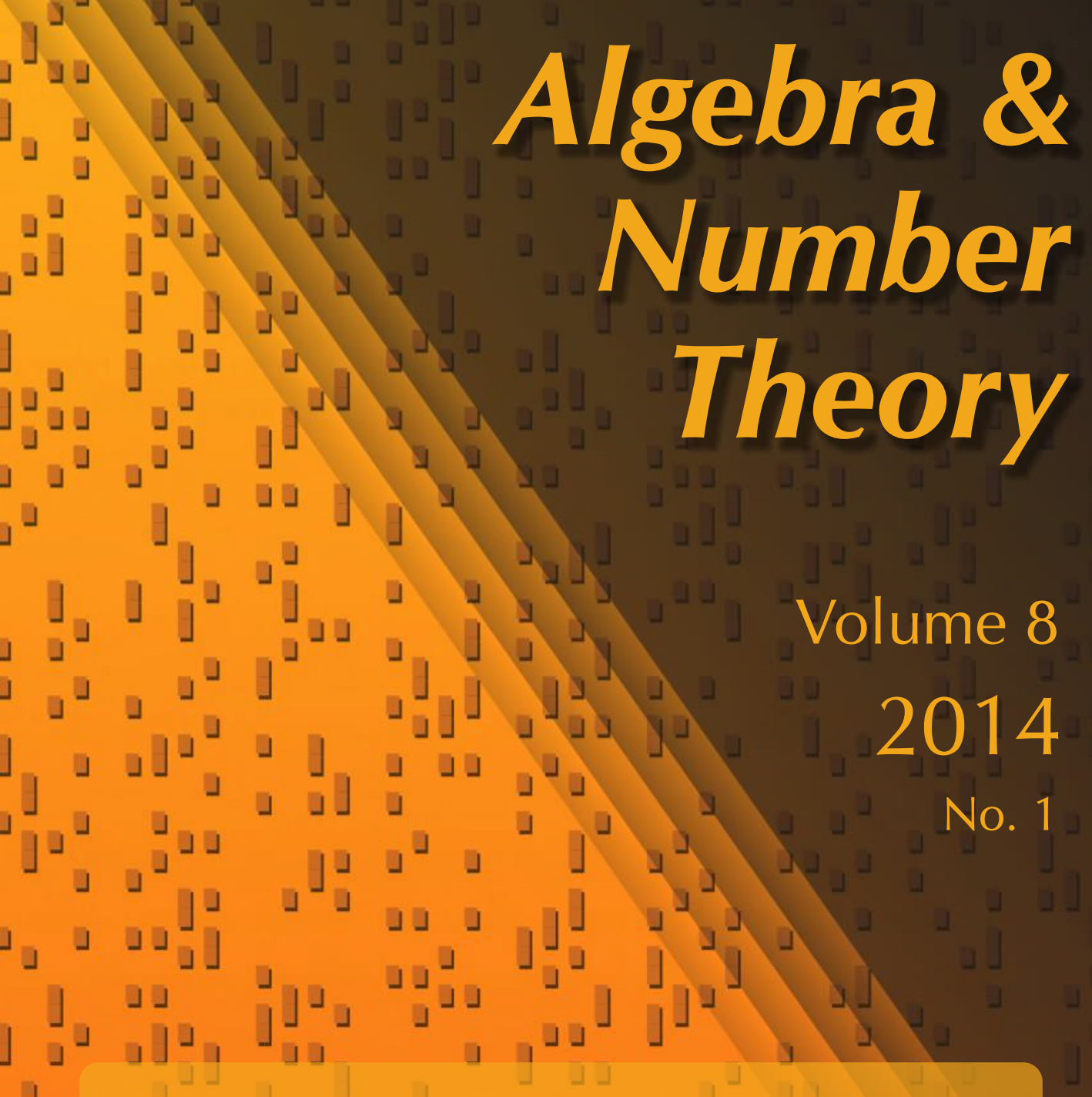

A generalized Bogomolov-Gieseker inequality for the three-dimensional projective space

4. Emanuele Macrì

\lrcorner

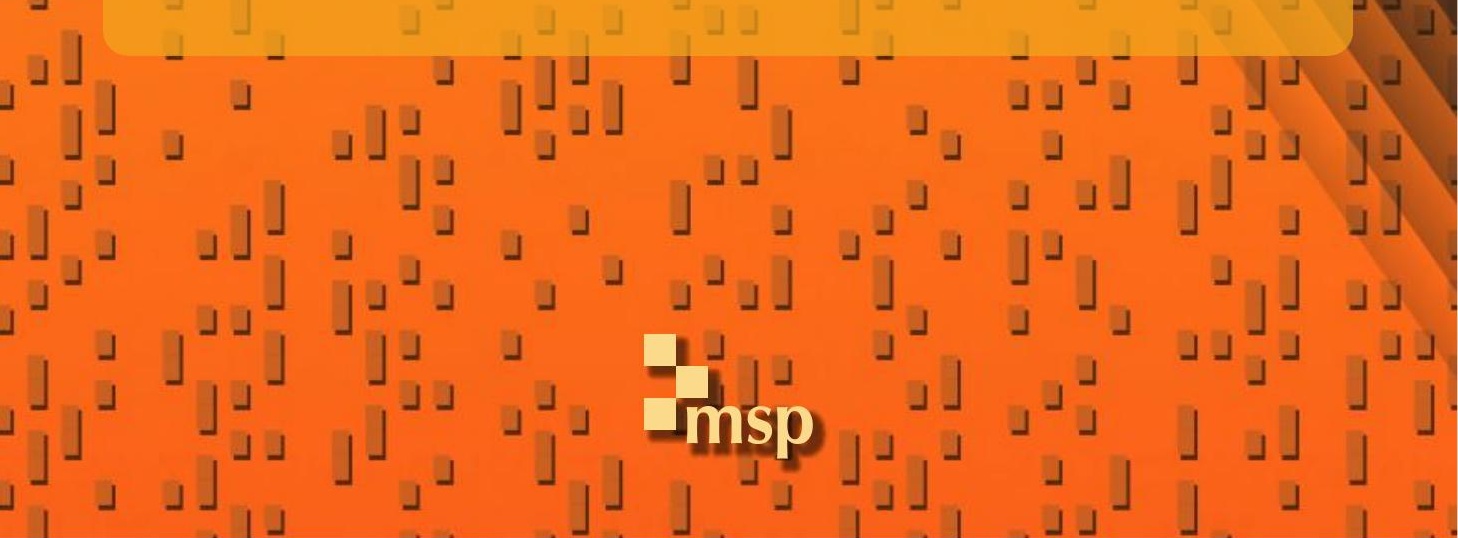




\title{
A generalized Bogomolov-Gieseker inequality for the three-dimensional projective space
}

\author{
Emanuele Macrì
}

\begin{abstract}
A generalized Bogomolov-Gieseker inequality for tilt-stable complexes on a smooth projective threefold was conjectured by Bayer, Toda, and the author. We show that such inequality holds true in general if it holds true when the polarization is sufficiently small. As an application, we prove it for the threedimensional projective space.
\end{abstract}

\section{Introduction}

The notion of tilt-stability, for objects in the derived category of a smooth projective threefold, was introduced in [Bayer et al. 2011b], based on [Bridgeland 2008; Arcara and Bertram 2013]. In [Bayer et al. 2011b, Conjecture 1.3.1] (Conjecture 2.3 of the present paper), we proposed a generalized Bogomolov-Gieseker inequality (BG inequality, for short) for tilt-stable objects. The main application for tilt-stability was to have an auxiliary notion of stability to construct Bridgeland stability conditions. The generalized BG inequality is precisely the missing ingredient to being able to show the existence of Bridgeland stability conditions.

In this note, we prove such inequality in the case of the projective space $\mathbb{P}^{3}$.

Theorem 1.1. The generalized Bogomolov-Gieseker inequality for tilt-stable objects in $\mathrm{D}^{\mathrm{b}}\left(\mathbb{P}^{3}\right)$ holds.

This gives the first example when the generalized BG inequality is proved in full generality. As a corollary, by [Bayer et al. 2011b], we can also describe a large open subset of the space of stability conditions on $\mathrm{D}^{\mathrm{b}}\left(\mathbb{P}^{3}\right)$. It would be very interesting to study how moduli spaces of Bridgeland semistable objects vary when varying the stability condition (very much like the situation described in [Arcara et al. 2013; Maciocia and Meachan 2013; Lo and Qin 2011; Minamide et al. 2011; Yanagida and Yoshioka 2012; Bayer and Macrì 2012; Toda 2012b; Yoshioka 2012] for the

MSC2010: primary 14F05; secondary 18E30, 14J30.

Keywords: Bridgeland stability conditions, derived category, Bogomolov-Gieseker inequality. 
case of surfaces). The behavior at the "large volume limit point" is described in [Bayer et al. 2011b, Section 6].

The idea of the proof of Theorem 1.1 goes as follows. For a smooth projective threefold $X$, the notion of tilt-stability depends on two parameters, namely two divisor classes $B, \omega \in \mathrm{NS}_{\mathbb{R}}(X)$ with $\omega$ ample. In this paper, we prove a general result, Proposition 2.7: showing the generalized BG inequality for all $B$ and $\omega$ can always be reduced to showing it for $\omega$ "arbitrarily small", uniformly in $B$.

For $X=\mathbb{P}^{3}$, the case in which $\omega$ is small was essentially proved in [Bayer et al. 2011b, Theorem 8.2.1]. More precisely, for simplicity, in [Bayer et al. 2011b], only the case $B=0$ was considered. Proposition 3.1 generalizes that argument to arbitrary $B$. Together with Proposition 2.7, this completes the proof of Theorem 1.1.

The interest for a general proof of the generalized BG inequality, besides for the existence of Bridgeland stability conditions, relies on its consequences. Indeed, if we assume such inequality to be true, we would have

- a proof of Fujita's conjecture for threefolds [Bayer et al. 2011a],

- a mathematical formulation of Denef and Moore's formula derived in the study of Ooguri, Strominger, and Vafa's conjecture, relating black-hole entropy and topological string [Toda 2013a], and

- the possibility to realize extremal contractions for threefolds as moduli spaces of semistable objects in the derived category [Toda 2013b].

We also mention that in the paper [Polishchuk 2012] the existence of Bridgeland stability conditions on abelian threefolds is tested on a class of objects (called Lagrangian-Invariant objects).

Finally, in [Bayer et al. 2011b], a strict relation between the generalized BG inequality and Castelnuovo's inequality for curves in $\mathbb{P}^{3}$ was pointed out. In Section 4 of this paper, we show that Theorem 1.1 gives, as an immediate corollary, a weaker version of Castelnuovo's theorem [Hartshorne 1977, IV, 6.4].

A survey on Bridgeland stability conditions and further problems and applications can be found in [Bridgeland 2009; Bayer 2011; Huybrechts 2012; Toda 2012a].

Notation. In this paper, we will always denote by $X$ a smooth projective threefold over the complex numbers and by $\mathrm{D}^{\mathrm{b}}(X)$ its bounded derived category of coherent sheaves. The Chow groups of $X$ modulo numerical equivalence are denoted by $\operatorname{Num}(X)$. In particular, the Néron-Severi group $\mathrm{NS}(X)=\operatorname{Num}^{1}(X)$. For an abelian group $G$ and a field $k(=\mathbb{Q}, \mathbb{R}, \mathbb{C})$, we denote by $G_{k}$ the $k$-vector space $G \otimes k$.

\section{The reduction argument}

In this section, we give a brief recall on the notion of tilt stability, following [Bayer et al. 2011b]. We show how to reduce the proof of the generalized BogomolovGieseker inequality proposed in [Bayer et al. 2011b, Conjecture 1.3.1] (whose 
statement is recalled in Conjecture 2.3 below), when $\omega$ and $B$ are "parallel", to the case in which the polarization is "sufficiently small".

2A. Tilt stability. Let $X$ be a smooth projective threefold over $\mathbb{C}$, and let $H \in \mathrm{NS}(X)$ be an ample divisor class. For a pair

$$
\begin{aligned}
& \omega=\alpha \cdot H, \quad \alpha \in \mathbb{R}_{>0}, \\
& B=\beta \cdot H, \quad \beta \in \mathbb{R},
\end{aligned}
$$

we define a slope function $\mu_{\omega, B}$ for coherent sheaves on $X$ in the usual way: for $E \in \operatorname{Coh}(X)$, we set

$$
\mu_{\omega, B}(E)=\left\{\begin{array}{cl}
+\infty & \text { if } \operatorname{ch}_{0}^{B}(E)=0, \\
\frac{\omega^{2} \operatorname{ch}_{1}^{B}(E)}{\omega^{3} \operatorname{ch}_{0}^{B}(E)} & \text { otherwise }
\end{array}\right.
$$

where $\operatorname{ch}^{B}(E)=e^{-B} \operatorname{ch}(E)$ denotes the Chern character twisted by $B$. Explicitly,

$$
\begin{array}{ll}
\operatorname{ch}_{0}^{B}=\mathrm{ch}_{0}, & \operatorname{ch}_{2}^{B}=\mathrm{ch}_{2}-B \mathrm{ch}_{1}+\frac{1}{2} B^{2} \mathrm{ch}_{0}, \\
\operatorname{ch}_{1}^{B}=\mathrm{ch}_{1}-B \mathrm{ch}_{0}, & \operatorname{ch}_{3}^{B}=\mathrm{ch}_{3}-B \mathrm{ch}_{2}+\frac{1}{2} B^{2} \mathrm{ch}_{1}-\frac{1}{6} B^{3} \mathrm{ch}_{0} .
\end{array}
$$

A coherent sheaf $E$ is slope-(semi)stable (or $\mu_{\omega, B^{-}}$(semi)stable) if, for all subsheaves $F \hookrightarrow E$, we have

$$
\mu_{\omega, B}(F)<(\leq) \mu_{\omega, B}(E / F) .
$$

Due to the existence of Harder-Narasimhan filtrations (HN-filtrations, for short) with respect to slope-stability, there exists a torsion pair $\left(\mathscr{T}_{\omega, B}, \mathscr{F}_{\omega, B}\right)$ defined as follows:

$$
\begin{aligned}
& \mathscr{T}_{\omega, B}=\left\{E \in \operatorname{Coh} X: \text { any quotient } E \rightarrow G \text { satisfies } \mu_{\omega, B}(G)>0\right\}, \\
& \mathscr{F}_{\omega, B}=\left\{E \in \operatorname{Coh} X: \text { any subsheaf } F \hookrightarrow E \text { satisfies } \mu_{\omega, B}(F) \leq 0\right\} .
\end{aligned}
$$

Equivalently, $\mathscr{T}_{\omega, B}$ and $\mathscr{F}_{\omega, B}$ are the extension-closed subcategories of $\operatorname{Coh} X$ generated by slope-stable sheaves of positive or nonpositive slope, respectively.

Definition 2.1. We let $\operatorname{Coh}^{\omega, B}(X) \subset \mathrm{D}^{\mathrm{b}}(X)$ be the extension-closure

$$
\operatorname{Coh}^{\omega, B}(X)=\left\langle\mathscr{T}_{\omega, B}, \mathscr{F}_{\omega, B}[1]\right\rangle .
$$

The category $\operatorname{Coh}^{\omega, B}(X)$ depends only on $\omega$ via $H$. Hence, to simplify notation, since for us $B$ is also a multiple of $H$, we denote it by $\operatorname{Coh}^{B}(X)$. By the general theory of torsion pairs and tilting [Happel et al. 1996], $\operatorname{Coh}^{B}(X)$ is the heart of a bounded $\mathrm{t}$-structure on $\mathrm{D}^{\mathrm{b}}(X)$. 
By using the classical Bogomolov-Gieseker inequality and Hodge index theorem, we can define the following slope function on $\operatorname{Coh}^{B}(X)$ : for $E \in \operatorname{Coh}^{B}(X)$, we set

$$
v_{\omega, B}(E)=\left\{\begin{array}{cl}
+\infty & \text { if } \omega^{2} \operatorname{ch}_{1}^{B}(E)=0, \\
\frac{\omega \operatorname{ch}_{2}^{B}(E)-\frac{1}{2} \omega^{3} \operatorname{ch}_{0}^{B}(E)}{\omega^{2} \operatorname{ch}_{1}^{B}(E)} & \text { otherwise. }
\end{array}\right.
$$

Definition 2.2. An object $E \in \operatorname{Coh}^{B}(X)$ is tilt-(semi)stable if, for all nontrivial subobjects $F \hookrightarrow E$, we have

$$
v_{\omega, B}(F)<(\leq) v_{\omega, B}(E / F) .
$$

The following is our main conjecture:

Conjecture 2.3 [Bayer et al. 2011b, Conjecture 1.3.1]. For any $v_{\omega, B}$-semistable object $E \in \operatorname{Coh}^{B}(X)$ satisfying $v_{\omega, B}(E)=0$, we have the following generalized Bogomolov-Gieseker inequality:

$$
\operatorname{ch}_{3}^{B}(E) \leq \frac{1}{6} \omega^{2} \operatorname{ch}_{1}^{B}(E) .
$$

The original definition of tilt-stability in [Bayer et al. 2011b] was given when $\alpha, \beta \in \mathbb{Q}$ (actually it was slightly more general, allowing $\omega$ and $B$ to be arbitrary, and $\omega$ had a different parametrization $\omega \mapsto \sqrt{3} \cdot \omega)$. The extension to $\mathbb{R}$ is the content of the following proposition, which we recall for later use:

Proposition 2.4 [Bayer et al. 2011b, Corollary 3.3.3]. Let $\operatorname{St}_{\mathrm{N}} \subset \mathrm{NS}_{\mathbb{R}}(X) \times \mathrm{NS}_{\mathbb{R}}(X)$ be the subset of pairs of real classes $(\omega, B)$ for which $\omega$ is ample. There exists a notion of "tilt-stability" for every $(\omega, B) \in \mathrm{St}$. For every object $E$, the set of $(\omega, B)$ for which $E$ is $v_{\omega, B}$-stable defines an open subset of $\mathrm{St}$.

Definition 2.5. We define the generalized discriminant

$$
\bar{\Delta}_{H}:=\left(H^{2} \operatorname{ch}_{1}^{B}\right)^{2}-2 H^{3} \operatorname{ch}_{0}^{B} \cdot\left(H \operatorname{ch}_{2}^{B}\right) .
$$

The generalized discriminant is independent of $\beta$. Indeed, by expanding the definition, we have

$$
\begin{aligned}
\bar{\Delta}_{H}= & \left(H^{2}\left(\operatorname{ch}_{1}-\beta \operatorname{ch}_{0} H\right)\right)^{2}-2 H^{3} \operatorname{ch}_{0} \cdot H\left(\operatorname{ch}_{2}-\beta H \operatorname{ch}_{1}+\frac{1}{2} \beta^{2} \operatorname{ch}_{0} H^{2}\right) \\
= & \left(H^{2} \operatorname{ch}_{1}\right)^{2}-2\left(H^{2} \operatorname{ch}_{1}\right) H^{3} \beta \operatorname{ch}_{0}+\beta^{2}\left(\operatorname{ch}_{0}\right)^{2}\left(H^{3}\right)^{2}-2 H^{3} \operatorname{ch}_{0}\left(H \operatorname{ch}_{2}\right) \\
& \quad+2\left(H^{2} \operatorname{ch}_{1}\right) H^{3} \beta \operatorname{ch}_{0}-\beta^{2}\left(\operatorname{ch}_{0}\right)^{2}\left(H^{3}\right)^{2} \\
= & \left(H^{2} \operatorname{ch}_{1}\right)^{2}-2 H^{3} \operatorname{ch}_{0}\left(H \operatorname{ch}_{2}\right) .
\end{aligned}
$$

The following result will be the key ingredient in our proof:

Theorem 2.6 [Bayer et al. 2011b, Corollary 7.3.2]. For any $v_{\omega, B}$-semistable object $E \in \operatorname{Coh}^{B}(X)$, we have

$$
\bar{\Delta}_{H}(E) \geq 0 \text {. }
$$


2B. Reduction to small $\omega$. In this section, we prove our reduction result. We keep the same notation as before, e.g., $\omega=\alpha H$ and $B=\beta H$. To simplify, we will write $v_{\alpha, \beta}$ for $v_{\omega, B}, \operatorname{Coh}^{\beta}(X)$, and so on.

Proposition 2.7. Assume there exists $\bar{\alpha} \in \mathbb{R}_{>0}$ such that, for all $\alpha<\bar{\alpha}$ and for all $\beta \in \mathbb{R}$, Conjecture 2.3 holds. Then Conjecture 2.3 holds for all $\alpha \in \mathbb{R}_{>0}$ and for all $\beta \in \mathbb{R}$.

To prove Proposition 2.7, we need first to introduce a bit more of notation. We denote by $\mathbb{W}$ the upper half-plane

$$
\mathbb{M}:=\left\{(\beta, \alpha) \in \mathbb{R}^{2}: \alpha>0\right\} .
$$

For a vector

$$
v:=\left(\mathrm{ch}_{0}, \mathrm{ch}_{1}, \mathrm{ch}_{2}, \mathrm{ch}_{3}\right) \in \operatorname{Num}_{\mathbb{Q}}(X)
$$

such that $H^{2} \operatorname{ch}_{1}^{\beta}>0$, the equation $v_{\alpha, \beta}(v)=0$ defines a curve $\mathscr{C}_{v}$ in $\mathbb{H}$. Explicitly, we have

$$
\mathscr{C}_{v}: H \mathrm{ch}_{2}-\beta\left(H^{2} \mathrm{ch}_{1}\right)+\frac{1}{2} \beta^{2} H^{3} \mathrm{ch}_{0}-\frac{1}{2} \alpha^{2} H^{3} \mathrm{ch}_{0}=0
$$

together with the inequality

$$
\beta H^{3} \operatorname{ch}_{0}<H^{2} \operatorname{ch}_{1} .
$$

We can divide into two cases:

$$
\begin{aligned}
& \operatorname{ch}_{0}=0 \rightsquigarrow \beta=\frac{H \mathrm{ch}_{2}}{H^{2} \mathrm{ch}_{1}}, \\
& \operatorname{ch}_{0} \neq 0 \rightsquigarrow\left(\beta-\frac{H^{2} \mathrm{ch}_{1}}{H^{3} \mathrm{ch}_{0}}\right)^{2}-\alpha^{2}=\frac{\bar{\Delta}_{H}}{\left(H^{3} \mathrm{ch}_{0}\right)^{2}} .
\end{aligned}
$$

Hence, if $\bar{\Delta}_{H} \geq 0$, then the tangent line at a point $\left(\beta_{0}, \alpha_{0}\right) \in \mathscr{C}$ intersects the line $\alpha=0$ with an angle $\pi / 4 \leq \theta \leq \pi / 2$.

Finally, on the curve $\mathscr{C}_{v}$, we can write the inequality (1) as follows:

$$
\begin{aligned}
& \operatorname{ch}_{0}=0 \rightsquigarrow \operatorname{ch}_{3}-\frac{\left(H \mathrm{ch}_{2}\right)^{2}}{2\left(H^{2} \mathrm{ch}_{1}\right)} \leq \alpha^{2} \frac{H^{2} \mathrm{ch}_{1}}{6}, \\
& \operatorname{ch}_{0} \neq 0 \rightsquigarrow \beta \frac{\bar{\Delta}_{H}}{H^{3} \operatorname{ch}_{0}} \leq \frac{\left(H \mathrm{ch}_{2}\right)\left(H^{2} \mathrm{ch}_{1}\right)}{H^{3} \operatorname{ch}_{0}}-3 \mathrm{ch}_{3} .
\end{aligned}
$$

Indeed, both inequalities in (4) follow directly by rewriting (1) by using (2) and (3), respectively.

Proof of Proposition 2.7. We argue by contradiction. Assume that there exist $\alpha_{0} \geq \bar{\alpha}$, $\beta_{0} \in \mathbb{R}$, and an object $E_{0} \in \mathrm{Coh}^{\beta_{0}}(X)$ that is $v_{\alpha_{0}, \beta_{0}}$-stable, such that $v_{\alpha_{0}, \beta_{0}}\left(E_{0}\right)=0$, and that does not satisfy the inequality in Conjecture 2.3. 


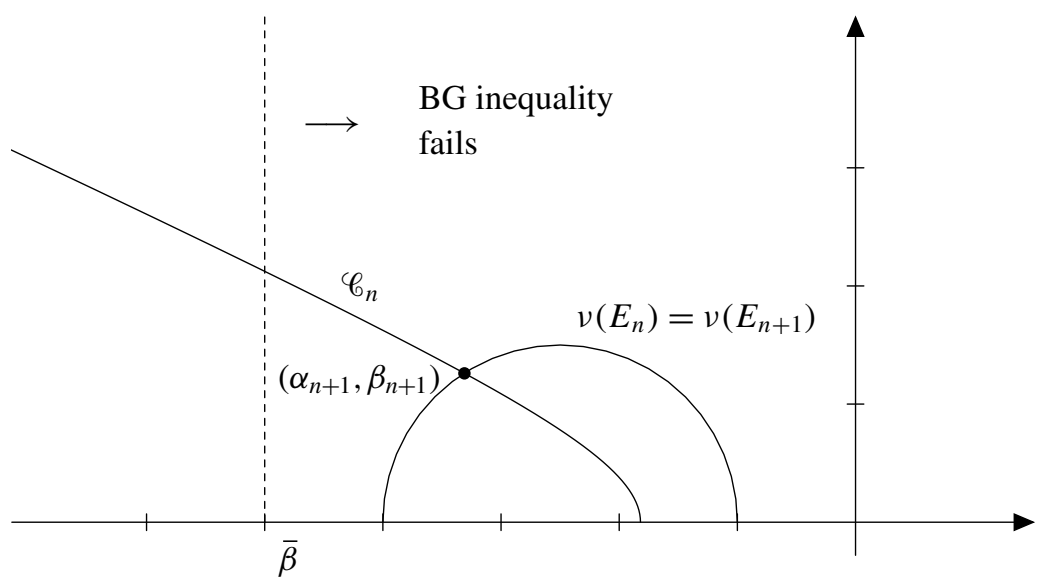

Figure 1. The curve $\mathscr{C}_{n}$ in the case $\operatorname{ch}_{0}\left(E_{n}\right)>0$. The BG inequality is not satisfied when $\beta>\bar{\beta}$, where $\bar{\beta}$ is defined in (5).

Claim 1. There exist a sequence $\left(\beta_{n}, \alpha_{n}\right) \in \mathbb{U}$ and a sequence of objects $\left\{E_{n}\right\}_{n \geq 0}$ such that

- $E_{n} \in \operatorname{Coh}^{\beta_{n}}(X) \cap \operatorname{Coh}^{\beta_{n+1}}(X)$ is $v_{\alpha_{n}, \beta_{n}}$-stable,

- $v_{\alpha_{n}, \beta_{n}}\left(E_{n}\right)=v_{\alpha_{n+1}, \beta_{n+1}}\left(E_{n}\right)=0$,

- $0<H^{2} \operatorname{ch}_{1}^{\beta_{n+1} H}\left(E_{n+1}\right)<H^{2} \operatorname{ch}_{1}^{\beta_{n+1} H}\left(E_{n}\right)$,

- $E_{n}$ does not satisfy the inequality (1),

- $\alpha_{0}>\alpha_{1}>\cdots>\alpha_{n}>\cdots>0$, and

- $\left|\beta_{n+1}\right| \leq\left|\beta_{0}\right|+\alpha_{0}$.

Proof. We proceed by induction, the case $n=0$ being our assumption. Assume that we have constructed $E_{n}$ with the wanted properties. By Proposition 2.4, the locus in $\mathbb{W}$ where $E_{n}$ is $v_{\alpha, \beta}$-stable is open. Consider the curve $\mathscr{C}:=\mathscr{C}_{\operatorname{ch}\left(E_{n}\right)} \subset \mathbb{U}$, and consider the set $U:=\left\{(\beta, \alpha) \in \mathscr{C}: \alpha<\alpha_{n}\right\}$. We claim that, for all $(\beta, \alpha) \in U$, the inequality (1) is not satisfied for $E_{n}$. Indeed, this can be seen by dividing into three cases, according to whether $\operatorname{ch}_{0}\left(E_{n}\right)$ is $>0,=0$, or $<0$ (the case in which $\operatorname{ch}_{0}\left(E_{n}\right)>0$ is illustrated in Figure 1). If $\operatorname{ch}_{0}\left(E_{n}\right)>0$, then by (4), we must have $\beta>\bar{\beta}$, where

$$
\bar{\beta}:=\frac{\left(H \mathrm{ch}_{2}\right)\left(H^{2} \mathrm{ch}_{1}\right)-3 H^{3} \mathrm{ch}_{0} \mathrm{ch}_{3}}{\bar{\Delta}_{H}} .
$$

But by assumption, $\beta<H^{2} \operatorname{ch}_{1}\left(E_{n}\right) / H^{3} \operatorname{ch}_{0}\left(E_{n}\right)$. Hence, the hyperbola $\mathscr{C}$ is decreasing, which is what we claimed. The case $\operatorname{ch}_{0}\left(E_{n}\right)<0$ is analogous, and the case $\operatorname{ch}_{0}\left(E_{n}\right)=0$ follows directly again from (4) since, in this case, $H^{2} \operatorname{ch}_{1}\left(E_{n}\right)>0$. 
Since Conjecture 2.3 holds when $\alpha<\bar{\alpha}$, there must exist $\left(\beta_{n+1}, \alpha_{n+1}\right) \in U$ such that $E_{n}$ is $v_{\alpha_{n+1}, \beta_{n+1}}$-semistable and is not $v_{\alpha, \beta}$-semistable for all $(\beta, \alpha) \in U$ with $\alpha<\alpha_{n+1}$. When $\operatorname{ch}_{0}\left(E_{n}\right) \neq 0$, the hyperbola $\mathscr{C}$ has asymptotes meeting at the point $\left(H^{2} \operatorname{ch}_{1}\left(E_{n}\right) / H^{3} \operatorname{ch}_{0}\left(E_{n}\right), 0\right)$. Hence, for all $(\beta, \alpha) \in U$, we must have $\beta H^{3} \operatorname{ch}_{0}\left(E_{n}\right)<H^{2} \operatorname{ch}_{1}\left(E_{n}\right)$. Therefore, $E_{n}$ being $v_{\alpha_{n+1}, \beta_{n+1}}$-semistable, it must belong to the category $\mathrm{Coh}^{\beta_{n+1}}(X)$.

By looking at the $v_{\alpha_{n+1}, \beta_{n+1}}$-stable factors of $E_{n}$ (by [Bayer et al. 2011b, Proposition 5.2.2], this makes sense in the category $\operatorname{Coh}^{\beta_{n+1}}(X)$ ), given the additivity of the Chern character, there must exist an object $E_{n+1} \in \mathrm{Coh}^{\beta_{n+1}}(X)$ that is $v_{\alpha_{n+1}, \beta_{n+1}}$ stable, such that $v_{\alpha_{n+1}, \beta_{n+1}}\left(E_{n+1}\right)=0$, and that does not satisfy the inequality (1).

The final inequality, $\left|\beta_{n+1}\right| \leq\left|\beta_{0}\right|+\alpha_{0}$, follows simply by the fact, observed before, that the tangent line at any point in $\mathscr{C}$ intersects the line $\alpha=0$ with an angle $\pi / 4 \leq \theta \leq \pi / 2$. See Figure 2 .

We let $\tilde{\alpha} \geq 0$ be the limit of the sequence $\left\{\alpha_{n}\right\}$. By assumption, we would get a contradiction if we prove that $\tilde{\alpha}=0$. Hence, assume this is not the case, namely $\tilde{\alpha}>0$. The idea is to find bounds for $\operatorname{ch}_{0}\left(E_{n}\right), H^{2} \operatorname{ch}_{1}\left(E_{n}\right)$, and $H \operatorname{ch}_{2}\left(E_{n}\right)$.

Claim 2. For all $n>0$, the following inequality holds:

$$
\bar{\Delta}_{H}\left(E_{n}\right)+\left(\alpha_{n} H^{3} \operatorname{ch}_{0}\left(E_{n}\right)\right)^{2}<\bar{\Delta}_{H}\left(E_{0}\right)+\left(\alpha_{0} H^{3} \operatorname{ch}_{0}\left(E_{0}\right)\right)^{2} .
$$

Proof. Again, we proceed by induction. By Claim 1, and by definition of the generalized discriminant, we have

$$
\begin{aligned}
\bar{\Delta}_{H} & \left(E_{n+1}\right)+\left(\alpha_{n+1} H^{3} \operatorname{ch}_{0}\left(E_{n+1}\right)\right)^{2} \\
& =\left(H^{2} \operatorname{ch}_{1}^{\beta_{n+1}}\left(E_{n+1}\right)\right)^{2}-2 H^{3} \operatorname{ch}_{0}\left(E_{n+1}\right)\left(H \mathrm{ch}_{2}^{\beta_{n+1}}\left(E_{n+1}\right)\right)+\left(\alpha_{n+1} H^{3} \operatorname{ch}_{0}\left(E_{n+1}\right)\right)^{2} \\
& =\left(H^{2} \operatorname{ch}_{1}^{\beta_{n+1}}\left(E_{n+1}\right)\right)^{2}-2 H^{3} \operatorname{ch}_{0}\left(E_{n+1}\right)\left(\frac{1}{2} \alpha_{n+1}^{2} H^{3} \operatorname{ch}_{0}\left(E_{n+1}\right)\right)+\left(\alpha_{n+1} H^{3} \operatorname{ch}_{0}\left(E_{n+1}\right)\right)^{2} \\
& =\left(H^{2} \operatorname{ch}_{1}^{\beta_{n+1}}\left(E_{n+1}\right)\right)^{2} \\
& <\left(H^{2} \operatorname{ch}_{1}^{\beta_{n+1}}\left(E_{n}\right)\right)^{2} \\
& =\left(H^{2} \operatorname{ch}_{1}^{\beta_{n+1}}\left(E_{n}\right)\right)^{2}-2 H^{3} \operatorname{ch}_{0}\left(E_{n}\right)\left(\frac{1}{2} \alpha_{n+1}^{2} H^{3} \operatorname{ch}_{0}\left(E_{n}\right)\right)+\left(\alpha_{n+1} H^{3} \operatorname{ch}_{0}\left(E_{n}\right)\right)^{2} \\
& =\left(H^{2} \operatorname{ch}_{1}^{\beta_{n+1}}\left(E_{n}\right)\right)^{2}-2 H^{3} \operatorname{ch}_{0}\left(E_{n}\right)\left(H \operatorname{ch}_{2}^{\beta_{n+1}}\left(E_{n}\right)\right)+\left(\alpha_{n+1} H^{3} \operatorname{ch}_{0}\left(E_{n}\right)\right)^{2} \\
& =\bar{\Delta}_{H}\left(E_{n}\right)+\left(\alpha_{n+1} H^{3} \operatorname{ch}_{0}\left(E_{n}\right)\right)^{2} \\
& \leq \bar{\Delta}_{H}\left(E_{n}\right)+\left(\alpha_{n} H^{3} \operatorname{ch}_{0}\left(E_{n}\right)\right)^{2} .
\end{aligned}
$$

By Claim 2, we deduce, for all $n>0$, the inequality

$$
\bar{\Delta}_{H}\left(E_{n}\right)+\left(\tilde{\alpha} H^{3} \operatorname{ch}_{0}\left(E_{n}\right)\right)^{2}<\bar{\Delta}_{H}\left(E_{0}\right)+\left(\alpha_{0} H^{3} \operatorname{ch}_{0}\left(E_{0}\right)\right)^{2} .
$$

Hence, we get immediately

$$
\bar{\Delta}_{H}\left(E_{n}\right)<\bar{\Delta}_{H}\left(E_{0}\right)+\left(\alpha_{0} H^{3} \operatorname{ch}_{0}\left(E_{0}\right)\right)^{2}=: \Gamma_{0},
$$




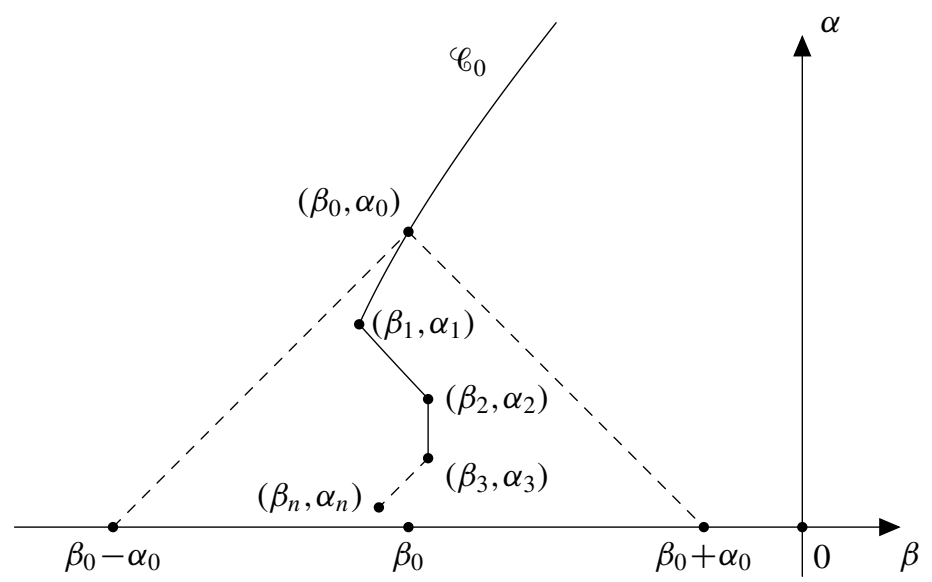

Figure 2. The sequence $\left(\beta_{n}, \alpha_{n}\right)$.

and by Theorem 2.6, we have

$$
\left(\operatorname{ch}_{0}\left(E_{n}\right)\right)^{2}<\frac{1}{\left(\tilde{\alpha} H^{3}\right)^{2}}\left(\bar{\Delta}_{H}\left(E_{0}\right)+\left(\alpha_{0} H^{3} \operatorname{ch}_{0}\left(E_{0}\right)\right)^{2}\right)=\Gamma_{1} .
$$

Finally, to bound $H^{2} \mathrm{ch}_{1}$, assume first that $\mathrm{ch}_{0}\left(E_{n}\right) \neq 0$. Then, by (3), (6), (7), and Claim 1, we have

$$
\left|H^{2} \operatorname{ch}_{1}\left(E_{n}\right)\right| \leq H^{3} \sqrt{\Gamma_{1}}\left(\left|\beta_{0}\right|+\alpha_{0}+\sqrt{\alpha_{0}^{2}+\frac{\Gamma_{0}}{\left(H^{3}\right)^{2}}}\right)=: \Gamma_{2} .
$$

The case in which $\operatorname{ch}_{0}\left(E_{n}\right)=0$ follows by Claim 1 by observing that either $\operatorname{ch}_{0}\left(E_{m}\right)=0$ for all $0 \leq m \leq n$ or there exists a maximum $0 \leq m<n$ for which $\operatorname{ch}_{0}\left(E_{m}\right) \neq 0$. In the first case, we have

$$
0<H^{2} \operatorname{ch}_{1}\left(E_{n}\right)<H^{2} \operatorname{ch}_{1}\left(E_{0}\right)
$$

while in the second

$$
0<H^{2} \operatorname{ch}_{1}\left(E_{n}\right)<\left|H^{2} \operatorname{ch}_{1}\left(E_{m}\right)\right|+\left|\beta_{m}\right|\left|\operatorname{ch}_{0}\left(E_{m}\right)\right| \leq \Gamma_{2}+\left(\left|\beta_{0}\right|+\alpha_{0}\right) \Gamma_{1} .
$$

Summing up, by (6), (7), (8), (9), and (10), we found bounds for $\operatorname{ch}_{0}\left(E_{n}\right)$, $H^{2} \operatorname{ch}_{1}\left(E_{n}\right)$, and $H \operatorname{ch}_{2}\left(E_{n}\right)$ for all $n$. But this shows that these classes are finite, and so there must exist an object $E$ that does not satisfy the inequality in Conjecture 2.3 for all $\alpha$ close to 0 , which contradicts our assumption.

\section{The case of the projective space}

In this section, we expand [Bayer et al. 2011b, Section 8.2] to show that, in the case of $X=\mathbb{P}^{3}$, the assumptions in Proposition 2.7 are satisfied. This will complete the 
proof of Theorem 1.1. To simplify notation, we directly identify $\operatorname{Num}_{\mathbb{R}}\left(\mathbb{P}^{3}\right)$ with $\mathbb{R}^{\oplus 4}$, and we take $\omega=\alpha, B=\beta \in \mathbb{R}$, and $\alpha>0$. The tilted slope becomes, up to an irrelevant multiplicative constant,

$$
v_{\alpha, \beta}=\frac{\operatorname{ch}_{2}^{\beta}-\frac{1}{2} \alpha^{2} \operatorname{ch}_{0}}{\operatorname{ch}_{1}^{\beta}}=\frac{\operatorname{ch}_{2}-\beta \operatorname{ch}_{1}+\left(\frac{1}{2} \beta^{2}-\frac{1}{2} \alpha^{2}\right) \operatorname{ch}_{0}}{\operatorname{ch}_{1}-\beta \operatorname{ch}_{0}} .
$$

Proposition 3.1. For all $\alpha<\frac{1}{3}$ and for all $\beta \in \mathbb{R}$, Conjecture 2.3 holds.

The proof is an adaptation of [Bayer et al. 2011b, Section 8.2], where only the case $\beta=0$ was considered. The idea is to use the existence of Bridgeland's stability conditions on $\mathrm{D}^{\mathrm{b}}\left(\mathbb{P}^{3}\right)$ associated to strong exceptional collections of sheaves (see [Bridgeland 2007, Example 5.5; Macrì 2007, Section 3.3)]. Here, we will use the full strong exceptional collection $\mathfrak{E}$ on $\mathrm{D}^{\mathrm{b}}\left(\mathbb{P}^{3}\right)$ given by

$$
\mathfrak{E}:=\left\{\mathcal{O}_{\mathbb{P}^{3}}(-1), 2, \mathscr{O}_{\mathbb{P}^{3}}, \mathscr{O}_{\mathbb{P}^{3}}(1)\right\},
$$

where $2:=T_{\mathbb{P}^{3}}(-2)$ is given by

$$
0 \rightarrow \mathrm{O}_{\mathbb{P}^{3}}(-2) \rightarrow \mathrm{O}_{\mathbb{P}^{3}}(-1)^{\oplus 4} \rightarrow 2 \rightarrow 0 .
$$

We consider the region $V$ given by

$$
V:=\left\{(\beta, \alpha) \in \mathbb{M}: 0 \geq \beta>-\frac{2}{3}, 0<\alpha<\frac{1}{3}\right\} .
$$

Lemma 3.2. Assume that Conjecture 2.3 holds for all $(\beta, \alpha) \in V$. Then it holds for all $\alpha<\frac{1}{3}$ and for all $\beta \in \mathbb{R}$.

Proof. Assume, for a contradiction, there exist $\alpha_{0}<\frac{1}{3}$ and $\beta_{0} \in \mathbb{R}$ and $E \in \mathrm{D}^{\mathrm{b}}\left(\mathbb{P}^{3}\right)$ that does not satisfy Conjecture 2.3. By acting with the autoequivalence $\otimes \mathbb{O}_{\mathbb{P}^{3}}(1)$ and with the local dualizing functor $\mathbb{D}(\cdot):=\boldsymbol{R} \mathscr{H} \mathrm{om}\left(\cdot, \mathrm{O}_{X}[1]\right)$, we can assume (see [Bayer et al. 2011b, Proposition 5.1.3]) that $0>\beta_{0} \geq-\frac{1}{2}$, which contradicts our assumption.

The next result will allow us to use the exceptional collection $\mathfrak{E}$ for doing computations. We postpone the proof to the end of the section.

Lemma 3.3. For all $(\beta, \alpha) \in V$, we have $2[1] \in \operatorname{Coh}^{\beta}\left(\mathbb{P}^{3}\right)$ and $v_{\alpha, \beta}^{\min }(2[1])>0$.

We divide the region $V$ into three parts:

$$
\begin{aligned}
& V_{1}:=\{(\beta, \alpha) \in V: \beta<-\alpha\}, \\
& V_{2}:=\{(\beta, \alpha) \in V: \beta>-\alpha\}, \\
& V_{3}:=\{(\beta, \alpha) \in V: \beta=-\alpha\} .
\end{aligned}
$$



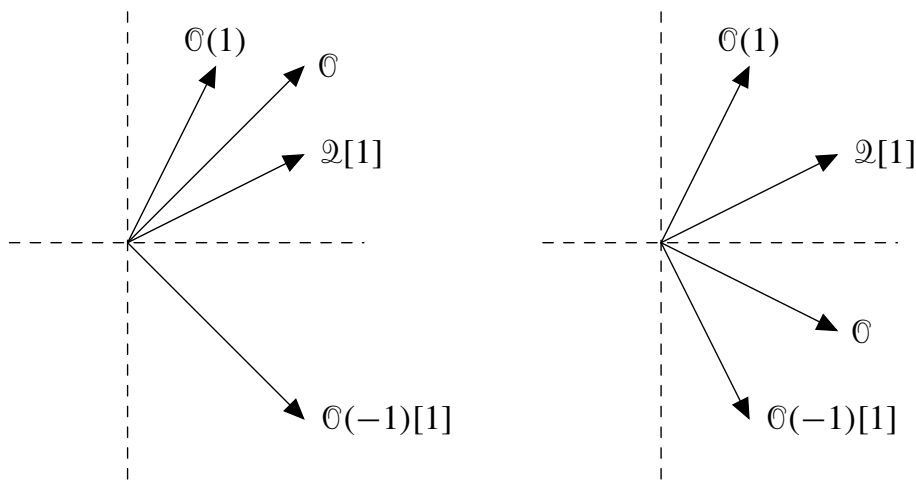

Figure 3. The slopes in $\mathrm{Coh}^{\beta}\left(\mathbb{P}^{3}\right)$ of the exceptional objects when $(\beta, \alpha) \in V_{1}$ (left) and $(\beta, \alpha) \in V_{2}$ (right). The tilt to $\mathscr{A}^{\alpha, \beta}$ corresponds to considering the upper half-plane. The two-dimensional picture is obtained by plotting denominator and numerator of $v_{\alpha, \beta}$. It is therefore oriented counterclockwise.

We first examine $V_{1}$ and $V_{2}$. On $V_{1}$, we have

$$
\begin{aligned}
v_{\alpha, \beta}(\mathbb{O}) & =\frac{1}{2} \cdot \frac{\beta^{2}-\alpha^{2}}{-\beta}>0, \\
v_{\alpha, \beta}(\mathbb{O}(-1)) & =\frac{1}{2} \cdot \frac{(\beta+1)^{2}-\alpha^{2}}{-\beta-1}<0, \\
v_{\alpha, \beta}(\mathcal{O}(1)) & =\frac{1}{2} \cdot \frac{(\beta-1)^{2}-\alpha^{2}}{1-\beta}>0, \\
v_{\alpha, \beta}(2) & =\frac{3}{2} \cdot \frac{\left(\beta+\frac{2}{3}\right)^{2}-\alpha^{2}-\frac{4}{9}}{-2-3 \beta}>0 .
\end{aligned}
$$

On $V_{2}$, we get the same expressions, but now $v_{\alpha, \beta}(\mathbb{O})<0$ (see Figure 3 ).

We now tilt one more time $\mathrm{Coh}^{\beta}\left(\mathbb{P}^{3}\right)$, as explained in [Bayer et al. 2011b, Definition 3.2.5]. As in Section 2A, we can define a torsion pair

$$
\begin{aligned}
& \mathscr{T}_{\omega, B}^{\prime}=\left\{E \in \operatorname{Coh}^{\beta}\left(\mathbb{P}^{3}\right): \text { any quotient } E \rightarrow G \text { satisfies } v_{\alpha, \beta}(G)>0\right\}, \\
& \mathscr{F}_{\omega, B}^{\prime}=\left\{E \in \operatorname{Coh}^{\beta}\left(\mathbb{P}^{3}\right): \text { any subsheaf } F \hookrightarrow E \text { satisfies } v_{\alpha, \beta}(F) \leq 0\right\} .
\end{aligned}
$$

We let $\mathscr{A}^{\alpha, \beta} \subset \mathrm{D}^{\mathrm{b}}\left(\mathbb{P}^{3}\right)$ be the extension-closure

$$
\mathscr{A}^{\alpha, \beta}:=\left\langle\mathscr{T}_{\alpha, \beta}^{\prime}, \mathscr{F}_{\alpha, \beta}^{\prime}[1]\right\rangle
$$



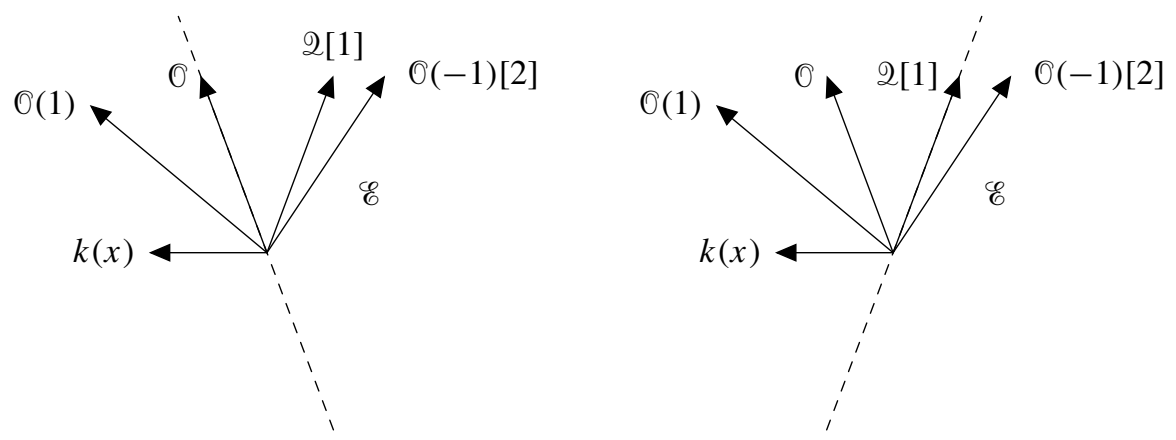

Figure 4. The slopes in $\mathscr{A}^{\alpha, \beta}$ of the exceptional objects and the skyscraper sheaves when $(\beta, \alpha) \in V_{1}$ (left) and $(\beta, \alpha) \in V_{2}$ (right). The category $\mathscr{E}$, obtained by tilting to the right along the dotted line, is the extension-closed subcategory generated by $\mathbb{O}(-1)[2]$, 2[1], $\mathbb{O}$, and $\mathcal{O}(1)[-1]$. It is equivalent to the category of modules over the finite-dimensional algebra determined by the dual exceptional collection to $\mathfrak{E}$.

By the previous computation, by [Bayer et al. 2011b, Proposition 7.4.1], and by Lemma 3.3, we have

$$
\begin{array}{ll}
\left\{\mathscr{O}_{\mathbb{P}^{3}}(-1)[2], 2[1], \mathscr{O}_{\mathbb{P}^{3}}, \mathcal{O}_{\mathbb{P}^{3}}(1)\right\} \subset \mathscr{A}^{\alpha, \beta} & \text { for }(\beta, \alpha) \in V_{1}, \\
\left\{\mathcal{O}_{\mathbb{P}^{3}}(-1)[2], 2[1], \mathscr{O}_{\mathbb{P}^{3}}[1], \mathcal{O}_{\mathbb{P}^{3}}(1)\right\} \subset \mathscr{A}^{\alpha, \beta} & \text { for }(\beta, \alpha) \in V_{2} .
\end{array}
$$

On the category $\mathscr{A}^{\alpha, \beta}$, we consider the following function (a posteriori, this will be a slope function):

$$
\lambda_{\alpha, \beta}:=\left\{\begin{array}{cl}
+\infty & \text { if } \operatorname{ch}_{2}^{\beta}-\frac{1}{2} \alpha^{2} \operatorname{ch}_{0}^{\beta}=0, \\
\frac{\operatorname{ch}_{3}^{\beta}-\frac{1}{6} \alpha^{2} \operatorname{ch}_{1}^{\beta}}{\operatorname{ch}_{2}^{\beta}-\frac{1}{2} \alpha^{2} \operatorname{ch}_{0}^{\beta}} & \text { otherwise. }
\end{array}\right.
$$

We have

$$
\begin{aligned}
\lambda_{\alpha, \beta}(\mathcal{O}) & =-\frac{1}{3} \beta \\
\lambda_{\alpha, \beta}(\mathcal{O}(-1)) & =-\frac{1}{3} \beta-\frac{1}{3}, \\
\lambda_{\alpha, \beta}(\mathcal{O}(1)) & =-\frac{1}{3} \beta+\frac{1}{3} \\
\lambda_{\alpha, \beta}(2) & =\frac{\left(\frac{2}{3}-\beta^{2}-\frac{1}{2} \beta^{3}\right)+\frac{1}{6} \alpha^{2}(3 \beta+2)}{2 \beta+\frac{3}{2} \beta^{2}-\frac{3}{2} \alpha^{2}} .
\end{aligned}
$$

On $V_{1}$, we deduce that $\lambda_{\alpha, \beta}(Q)<\lambda_{\alpha, \beta}(\mathcal{O}(1))$ while, on $V_{2}, \lambda_{\alpha, \beta}(Q)<\lambda_{\alpha, \beta}(\mathbb{O})$ (see Figure 4). 
By [Bayer et al. 2011b, Proposition 8.1.1] (and mimicking the proof of [Bayer et al. 2011b, Theorem 8.2.1]), this shows that Conjecture 2.3 holds for all $(\beta, \alpha) \in$ $V_{1} \cup V_{2}$.

To deal with the region $V_{3}$ (namely, the case $\alpha=-\beta$ ), we consider a slightly modified function on $\mathscr{A}^{\alpha, \beta}$

$$
\lambda_{\alpha, \beta}:=\left\{\begin{array}{cl}
+\infty & \text { if } \operatorname{ch}_{2}^{\beta}-\frac{1}{2} \alpha^{2} \operatorname{ch}_{0}^{\beta}=0, \\
\frac{\operatorname{ch}_{3}^{\beta}-\frac{1}{6} \alpha^{2} \operatorname{ch}_{1}^{\beta}-\epsilon \operatorname{ch}_{1}^{\beta}}{\operatorname{ch}_{2}^{\beta}-\frac{1}{2} \alpha^{2} \operatorname{ch}_{0}^{\beta}} & \text { otherwise, }
\end{array}\right.
$$

where $\epsilon>0$. In this case, we still have

$$
\left\{\mathscr{O}_{\mathbb{P}^{3}}(-1)[2], 2[1], \mathscr{O}_{\mathbb{P}^{3}}[1], \mathscr{O}_{\mathbb{P}^{3}}(1)\right\} \subset \mathscr{A}^{\alpha, \beta},
$$

and

$$
\begin{aligned}
\lambda_{\alpha, \beta}(\mathbb{O}) & =+\infty, \\
\lambda_{\alpha, \beta}(\mathbb{O}(-1)) & =-\frac{1}{3} \beta-\frac{1}{3}+2 \epsilon \frac{\beta+1}{2 \beta+1}, \\
\lambda_{\alpha, \beta}(\mathcal{O}(1)) & =-\frac{1}{3} \beta+\frac{1}{3}+2 \epsilon \frac{\beta-1}{1-2 \beta}, \\
\lambda_{\alpha, \beta}(2) & =\frac{1-\beta^{2}}{3 \beta}+\epsilon \frac{3 \beta+2}{2 \beta} .
\end{aligned}
$$

We deduce that, for all $0>\beta>-\frac{1}{3}$, there exists $\epsilon(\beta)>0$ such that

$$
\lambda_{\alpha, \beta}(\mathbb{O}(1))>\lambda_{\alpha, \beta}(\mathbb{O}(-1)) \quad \text { and } \quad \lambda_{\alpha, \beta}(\mathscr{O}(1))>\lambda_{\alpha, \beta}(2)
$$

when $(\beta, \alpha) \in V_{3}$ and $\epsilon<\epsilon(\beta)$. Again, by [Bayer et al. 2011b, Proposition 8.1.1], if we fix $\beta$ and let $\epsilon \rightarrow 0$, this shows that Conjecture 2.3 holds also for all $(\beta, \alpha) \in V_{3}$. By Lemma 3.2, this would complete the proof of Proposition 3.1 once Lemma 3.3 is proved.

Proof of Lemma 3.3. Since $2 \in \operatorname{Coh}\left(\mathbb{P}^{3}\right)$ is slope-stable with Chern character $\operatorname{ch}(2)=\left(3,-2,0, \frac{2}{3}\right)$, we have, by definition, $2[1] \in \operatorname{Coh}^{\beta}\left(\mathbb{P}^{3}\right)$ for all $\beta \geq-\frac{2}{3}$. Moreover, for $0 \geq \beta>-\frac{2}{3}$ and for all $\alpha>0$, we have $v_{\alpha, \beta}(2[1])>0$.

Assume, for a contradiction, there exists $\left(\beta_{0}, \alpha_{0}\right) \in V$ such that $v_{\alpha_{0}, \beta_{0}}^{\min }(Q[1]) \leq 0$. Let $N_{0} \in \mathrm{Coh}^{\beta_{0}}\left(\mathbb{P}^{3}\right)$ be the tilt-stable quotient $2[1] \rightarrow N_{0}$ in $\operatorname{Coh}^{\beta_{0}}\left(\mathbb{P}^{3}\right)$ such that $v_{\alpha_{0}, \beta_{0}}\left(N_{0}\right) \leq 0$. By taking the long exact sequence in cohomology, $N_{0} \cong M_{0}[1]$, where $M_{0} \in \operatorname{Coh}\left(\mathbb{P}^{3}\right)$ is a torsion-free sheaf.

Consider the curves $\mathscr{P}_{0}$, given by $v_{\alpha, \beta}(2[1])=v_{\alpha, \beta}\left(N_{0}\right)$, and $\mathscr{C}_{0}$, given by $v_{\alpha, \beta}\left(N_{0}\right)=0$ in the region $\beta>\operatorname{ch}_{1}\left(M_{0}\right) / \operatorname{ch}_{0}\left(M_{0}\right)$. Since the vector $(3,-2,0)$ is

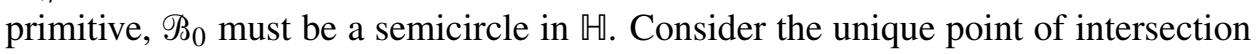
$(x, y) \in \mathscr{C}_{0} \cap \mathscr{P}_{0}$. Since $v_{\alpha, \beta}(2[1])>0$, for $0 \geq \beta>-\frac{2}{3}$, we have $x>0$. In particular, $\mathscr{B}_{0} \cap\{\beta=0\} \neq \varnothing$. See Figure 5 . 


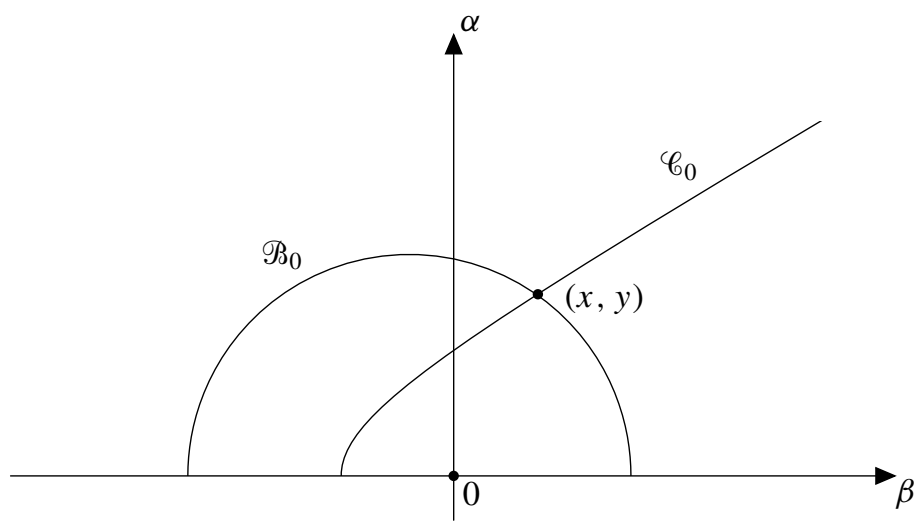

Figure 5. The curves $\mathscr{B}_{0}$ and $\mathscr{C}_{0}$.

By Bertram's nested wall theorem of [Maciocia 2012] (whose proof works as well in our context due to Theorem 2.6), we know that pseudo-walls for 2[1] are nested semicircles; namely, either $2[1]$ is tilt-stable outside $\mathscr{B}_{0}$ and unstable in the interior, or there exists another semicircle $\mathscr{B}_{1}$ with the same property and $\mathscr{P}_{1}$ contains $\mathscr{B}_{0}$ in its interior. In both cases, by the previous argument, the semicircles $\mathscr{B}_{0}$ and $\mathscr{B}_{1}$ intersect the half-line $\beta=0$. Hence, there exists $\alpha_{1}>0$ such that 2[1] is not $v_{\alpha_{1}, 0}$-stable. This contradicts Lemma 3.4 below.

Lemma 3.4. For all $\alpha>0$, 2[1] is $v_{\alpha, 0}$-stable.

Proof. First of all, we observe that 2[1] is PGL(4)-invariant. By uniqueness of Harder-Narasimhan filtrations, if 2[1] is not tilt-stable, then its HN factors have to be PGL(4)-invariant as well.

Consider the category $\operatorname{Coh}^{\beta=0}\left(\mathbb{P}^{3}\right)$. The function $f_{0}:=\mathrm{ch}_{1}$ is additive and takes nonnegative integral values on $\operatorname{Coh}^{0}\left(\mathbb{P}^{3}\right)$. Since $f_{0}(2[1])=2$, if there exists an exact sequence in $\operatorname{Coh}^{0}\left(\mathbb{P}^{3}\right)$

$$
0 \rightarrow P \rightarrow 2[1] \rightarrow N \cong M[1] \rightarrow 0
$$

that is destabilizing with $N$ tilt-semistable, then $f_{0}(P)=f_{0}(N)=1$ and both $P$ and $N$ must be tilt-stable. To prove this claim, we first observe that 2 [1] cannot have any subobject $P$ with $f_{0}(P)=0$. Indeed, in such a case, by definition, $P$ belongs to the category generated by extensions by $F[1]$, where $F$ is a $\mu$-stable torsionfree sheaves with $\mu(F)=0$, and by torsion sheaves supported in dimension $\leq 1$. Therefore, $\operatorname{Hom}(P, 2[1])=0$. Hence, a subobject $P$ of 2[1] can have either $f_{0}(P)=1$ or $f_{0}(P)=2$. But if $f_{0}(P)=2$, then the sequence is not destabilizing. The same argument shows that $P$ and $N$ are also tilt-stable. 
The long exact sequence in cohomology gives

$$
0 \rightarrow \mathscr{H}^{-1}(P) \rightarrow 2 \rightarrow M \rightarrow \mathscr{H}^{0}(P) \rightarrow 0
$$

with $\mathscr{H}^{-1}(P)$ and $M$ torsion-free with $\mu_{\alpha, 0}^{\max } \leq 0$. Since (11) is destabilizing and both $P$ and $N$ are tilt-stable with $f_{0}=1$, we must have $\mu_{\alpha, 0}^{\max }(M), \mu_{\alpha, 0}^{\max }\left(\mathscr{H}^{-1}(P)\right)<0$. This shows that there are only two possibilities:

(a) either $\operatorname{ch}_{1}(M)=\operatorname{ch}_{1}\left(\mathscr{H}^{-1}(P)\right)=-1$,

(b) or $\mathscr{H}^{-1}(P)=0$.

For case (a), we must have $\operatorname{ch}_{1}\left(\mathscr{H}^{0}(P)\right)=0$, and so $\mathscr{H}^{0}(P)$ is a torsion sheaf supported on a one-dimensional subscheme. By the PGL(4)-invariance, $\mathscr{H}^{0}(P)=0$. Finally, since 2 is slope-stable, we must have $\operatorname{ch}_{0}\left(\mathscr{H}^{-1}(P)\right)=1$, and so $\mathscr{H}^{-1}(P) \cong$ $\Phi_{C}(-1)$ for $C \subset \mathbb{P}^{3}$ a one-dimensional subscheme of degree $d \geq 0$. Again, by the PGL(4)-invariance, $C=0$. Summarizing, we proved that in case (a), $P \cong$ $\mathcal{O}_{\mathbb{P}^{3}}(-1)[1]$. But then, the equation $v_{\alpha, 0}(2[1])=v_{\alpha, 0}(P)$ has no solutions, and so (11) cannot be destabilizing.

For case (b), we have $P \in \operatorname{Coh}\left(\mathbb{P}^{3}\right)$ and an exact sequence in $\operatorname{Coh}\left(\mathbb{P}^{3}\right)$

$$
0 \rightarrow 2 \rightarrow M \rightarrow P \rightarrow 0
$$

with $\operatorname{ch}_{1}(M)=-1, \operatorname{ch}_{1}(P)=1$, and $\operatorname{ch}_{0}(M) \geq 3$. We now use Theorem 2.6 once more. Indeed, since $N$ must be tilt-stable, we have

$$
\operatorname{ch}_{2}(M) \leq \frac{1}{2 \operatorname{ch}_{0}(M)}
$$

and so $\operatorname{ch}_{2}(M) \leq 0$. As a consequence, the equation $v_{\alpha, 0}(2[1])=v_{\alpha, 0}(P)$ has no solutions $\alpha>0$, and so (11) cannot be destabilizing also in this case.

\section{An application}

In this section, we briefly discuss an application of Theorem 1.1 and some examples.

In [Bayer et al. 2011b, Example 7.2.4], we pointed out a relation between Conjecture 2.3 and Castelnuovo's inequality for curves in $\mathbb{P}^{3}$. In particular, by using Castelnuovo's inequality, we showed that Conjecture 2.3 holds for ideal sheaves of curves with respect to some tilt-stability. It is interesting to observe that a sort of converse holds: from Theorem 1.1, we can deduce a certain inequality for curves in $\mathbb{P}^{3}$, which is much weaker than Castelnuovo's one but already nontrivial.

Corollary 4.1. Let $C$ be a pure one-dimensional scheme in $\mathbb{P}^{3}$ of degree $d \geq 2$. Let $h:=\operatorname{ch}_{3}\left(\Phi_{C}\right)-2 d$. Then

$$
h \leq \frac{2 d^{2}-5 d}{3} .
$$


Moreover, if $C$ is integral and not contained in a plane, then

$$
h \leq \frac{d^{2}-4 d}{3} .
$$

We recall that, for an ideal sheaf $\Im_{C}$ of an integral curve $C \subset \mathbb{P}^{3}$ of degree $d$ and arithmetic genus $g, h=g-1$. Hence, the inequality (13) compares with [Hartshorne 1977, IV, 6.4].

To prove Corollary 4.1, we introduce some more notation. We define the two semicircles

$$
\begin{aligned}
& \mathscr{B}_{1}: \alpha^{2}+\left(\beta+\frac{2 d+1}{2}\right)^{2}=\left(\frac{2 d-1}{2}\right)^{2}, \\
& \mathscr{B}_{2}: \alpha^{2}+\left(\beta+\frac{d+2}{2}\right)^{2}=\left(\frac{d-2}{2}\right)^{2} .
\end{aligned}
$$

They correspond to the loci

$$
v_{\alpha, \beta}\left(\mathscr{I}_{C}\right)=v_{\alpha, \beta}\left(\mathscr{O}_{\mathbb{P}^{3}}(-1)\right) \quad \text { and } \quad v_{\alpha, \beta}\left(\mathscr{I}_{C}\right)=v_{\alpha, \beta}\left(\mathbb{O}_{\mathbb{P}^{3}}(-2)\right),
$$

respectively. More generally, for an object $A \in \mathrm{D}^{\mathrm{b}}\left(\mathbb{P}^{3}\right)$ such that $\left(\operatorname{ch}_{0}(A), \operatorname{ch}_{1}(A)\right.$, $\left.\operatorname{ch}_{2}(A)\right)$ is not a multiple of $(1,0,-d)$, we denote by $\mathscr{B}_{A}$ the semicircle with equation $v_{\alpha, \beta}\left(\Phi_{C}\right)=v_{\alpha, \beta}(A)$.

Finally, as in Section $2 \mathrm{~B}$, we denote by $\mathscr{C}$ the branch of the hyperbola $v_{\alpha, \beta}\left(\Phi_{C}\right)=0$ in $\mathbb{U}$; explicitly,

$$
\mathscr{C}: \beta^{2}-\alpha^{2}=2 d, \quad \beta<0 .
$$

Proof of Corollary 4.1. For the first part of the statement, we would like to show that on the exterior part of the semicircle $\mathscr{B}_{1}$ in $\mathbb{H} \cap\{-2 d<\beta<-1\}$ the ideal sheaf $\mathscr{I}_{C}$ is $v_{\alpha, \beta}$-stable.

First of all, we consider the half-line $\beta=-1$ and the category $\operatorname{Coh}^{\beta=-1}\left(\mathbb{P}^{3}\right)$. The function $f_{-1}:=\mathrm{ch}_{1}+\mathrm{ch}_{0}$ is additive and takes nonnegative integral values on $\mathrm{Coh}^{-1}\left(\mathbb{P}^{3}\right)$. Since $f_{-1}\left(\Phi_{C}\right)=1$, then $\Phi_{C}$ must be $v_{\alpha,-1}$-stable for all $\alpha>0$.

We now consider the half-line $\beta=-2$ and the category $\mathrm{Coh}^{-2}\left(\mathbb{P}^{3}\right)$. By [Bridgeland 2008, Proposition 14.2] (whose proof generalizes to our case), we know that, for $\alpha \gg 0, \mathscr{I}_{C}$ is $v_{\alpha,-2}$-stable. Assume that $\mathscr{I}_{C}$ is not $v_{\alpha,-2}$-semistable for all $\alpha>0$. Then, by Proposition 2.4, there exists $\alpha_{0}>0$ such that $\Phi_{C}$ is $v_{\alpha,-2}$-stable

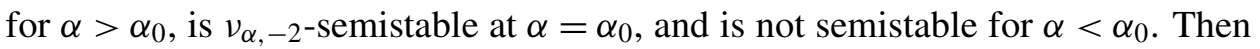
$\alpha_{0}$ must be in the intersection of the half-line $\beta=-2$ with a semicircle $\mathscr{B}_{A}$ for some $A \in \mathrm{Coh}^{-2}\left(\mathbb{P}^{3}\right)$ such that $A \hookrightarrow \Phi_{C}$ in $\operatorname{Coh}^{-2}\left(\mathbb{P}^{3}\right)$. By looking at the long exact sequence in cohomology, we deduce that $A \in \operatorname{Coh}\left(\mathbb{P}^{3}\right)$ and $\operatorname{ch}_{0}(A) \geq 1$ and it is torsion-free. Moreover, since the function $f_{-2}:=\operatorname{ch}_{1}+2 \mathrm{ch}_{0}$ is additive and takes nonnegative integral values on $\operatorname{Coh}^{-2}\left(\mathbb{P}^{3}\right)$ and $f_{-2}\left(\Phi_{C}\right)=2$, we must have 
$f_{-2}(A)=1$, namely

$$
\frac{\operatorname{ch}_{1}(A)}{\operatorname{ch}_{0}(A)}=-2+\frac{1}{\operatorname{ch}_{0}(A)} .
$$

Let $\left(-2, \alpha_{1}\right)$ be the intersection point in $\mathbb{H}$ between $\beta=-2$ and $\mathscr{B}_{1}$ (the intersection is nonempty since $d \geq 2$ ). We claim that $\alpha_{0} \leq \alpha_{1}$. Indeed, if $\operatorname{ch}_{0}(A)=1$, then $\operatorname{ch}_{1}(A)=-1$. Hence, $A \cong \mathscr{I}_{W}(-1)$ for some subscheme $W$ of dimension 1 . Therefore, $\alpha_{0} \leq \alpha_{1}$. If $\operatorname{ch}_{0}(A) \geq 2$, then $-2<\operatorname{ch}_{1}(A) / \operatorname{ch}_{0}(A)<-1$. By Bertram's nested wall theorem of [Maciocia 2012], we know that either $\mathscr{B}_{A}=\mathscr{B}_{1}$ or they are disjoint. Since $\mathscr{B}_{A} \cap\left\{\beta=\operatorname{ch}_{1}(A) / \operatorname{ch}_{0}(A)\right\}=\varnothing$, this immediately implies that $\alpha_{0} \leq \alpha_{1}$, as we wanted.

By using the nested wall theorem again, since we proved that, on the line $\beta=-1$, the ideal sheaf $\mathscr{I}_{C}$ is stable and, on the line $\beta=-2$, the first wall is $\mathscr{B}_{1}$, this shows that on the exterior part of the semicircle $\mathscr{B}_{1}$ in $\mathbb{H} \cap\{-2 d<\beta<-1\}$ the ideal sheaf $\Phi_{C}$ is $v_{\alpha, \beta}$-stable, which is what we wanted. To get the inequality (12), we only need to compute the intersection point $\mathscr{C} \cap \mathscr{B}_{1}$. Theorem 1.1 yields then directly (12).

The proof of (13) is very similar. We consider the half-line $\beta=-3$, the category $\mathscr{A}_{-3}:=\mathrm{Coh}^{-3}\left(\mathbb{P}^{3}\right)$, and $A \hookrightarrow \mathscr{I}_{C}$ in $\mathrm{Coh}^{-3}\left(\mathbb{P}^{3}\right)$. By looking at the function $f_{-3}:=$ $\mathrm{ch}_{1}+3 \mathrm{ch}_{0}$, we must have either $f_{-3}(A)=1$ or $=2$. If $\operatorname{ch}_{0}(A) \geq 3$, then by using again [Maciocia 2012], we can deduce that $\mathscr{B}_{A}$ is contained in the interior of $\mathscr{B}_{2}$. If $\operatorname{ch}_{0}(A)=2$, we distinguish two possibilities according to whether $f_{-3}(A)=1$ or $=2$. If $=1$, then we can argue as before and deduce that $\mathscr{P}_{A}$ is contained in the interior of $\mathscr{B}_{2}$. If $=2$, then $\operatorname{ch}_{1}(A)=-4$, and so by Theorem $2.6, \operatorname{ch}_{2}(A) \leq 4$. If $\operatorname{ch}_{2}(A)=4$, then $\mathscr{B}_{A}=\mathscr{B}_{2}$. If $\operatorname{ch}_{2}(A)<4$, then $\mathscr{B}_{A}$ is again contained in the interior of $\mathscr{B}_{2}$.

Finally, if $\operatorname{ch}_{0}(A)=1$, then either $A \cong I_{W}(-2)$ or $A \cong I_{W}(-1)$ with $W$ a closed subscheme of dimension 1 . The first case can be dealt as before. To exclude the second case, we use the assumption that $C$ is integral and not contained in a plane. Indeed, in such a case, we must have $C \subset W$, and so $A \hookrightarrow \Phi_{C}$ does not destabilize.

As before, to get the inequality (13), we only need to compute the intersection point $\mathscr{C} \cap \mathscr{B}_{2}$ and apply Theorem 1.1.

Example 4.2. For the case $d=1$, the situation is slightly degenerate. Indeed, in such a case, $\mathscr{I}_{C}$ is $v_{\alpha, \beta}$-semistable for all $(\beta, \alpha) \in \mathbb{W}$ for which

$$
\alpha^{2}+\left(\beta+\frac{3}{2}\right)^{2} \geq \frac{1}{4} .
$$

Hence, in particular, it is semistable for all $(\beta, \alpha) \in \mathscr{C}$. Theorem 1.1 gives then $h \leq-\frac{2}{3}$, namely $g(=0) \leq \frac{1}{3}$.

Example 4.3. If the curve $C$ in Corollary 4.1 is contained in a surface $F \subset \mathbb{P}^{3}$ of degree $k>0$, then there is a strong form for Castelnuovo's theorem, as proved by Harris [1980; Hartshorne 1978]. But in this case, we cannot directly conclude such inequality by using stability since it is not true that the first wall when $\mathscr{I}_{C}$ is 
destabilized coincides with the locus

$$
v_{\alpha, \beta}\left(\mathscr{I}_{C}\right)=v_{\alpha, \beta}\left(\mathbb{O}_{\mathbb{P}^{3}}(-k)\right), \quad \text { namely } \mathbb{O}_{\mathbb{P}^{3}}(-k) \hookrightarrow \mathscr{I}_{C} .
$$

The simplest example (see [Hartshorne 1977, V, 4.13.1]) is when $C$ is smooth with $k=3, d=7$, and $g=5$. In such a case, a destabilizing quotient is given instead by

$$
\Phi_{C} \rightarrow \mathcal{O}_{\mathbb{P}^{3}}(-5)[1] .
$$

This gives the (well-known) existence of a nontrivial extension, $\mathscr{G} \in \operatorname{Coh}\left(\mathbb{P}^{3}\right)$ of rank 2 , which must be stable. It may be interesting to study the general situation and see which kind of new stable objects arise as destabilizing factors of $\mathscr{I}_{C}$.

\section{Acknowledgements}

I would like to thank Arend Bayer and Ciaran Meachan for very useful discussions and Roman Bezrukavnikov for pointing out the idea of using Theorem 1.1 to show a version of Castelnuovo's theorem. I am also very grateful to the referee for the careful reading of the manuscript and for several suggestions that improved readability. This paper was completed during my stay at the Mathematical Institute of the University of Bonn, whose warm hospitality is gratefully acknowledged. I was partially supported by the NSF grant DMS-1160466 and, during the visit to Bonn, by the grant SFB/TR 45 .

\section{References}

[Arcara and Bertram 2013] D. Arcara and A. Bertram, "Bridgeland-stable moduli spaces for $K$-trivial surfaces", J. Eur. Math. Soc. 15:1 (2013), 1-38. MR 2998828 Zbl 1259.14014

[Arcara et al. 2013] D. Arcara, A. Bertram, I. Coskun, and J. Huizenga, "The minimal model program for the Hilbert scheme of points on $\mathbb{P}^{2}$ and Bridgeland stability", Adv. Math. 235 (2013), 580-626. MR 3010070 Zbl 1267.14023

[Bayer 2011] A. Bayer, "A tour to stability conditions on derived categories", notes, 2011, Available at http://www.maths.ed.ac.uk/ abayer/dc-lecture-notes.pdf.

[Bayer and Macrì 2012] A. Bayer and E. Macrì, "Projectivity and birational geometry of Bridgeland moduli spaces", preprint, 2012. To appear in J. Am. Math. Soc. arXiv 1203.4613v1

[Bayer et al. 2011a] A. Bayer, A. Bertram, E. Macrì, and Y. Toda, "Bridgeland stability conditions on threefolds, II: An application to Fujita's conjecture", preprint, 2011. To appear in J. Alg. Geom. arXiv 1106.3430v1

[Bayer et al. 2011b] A. Bayer, E. Macrì, and Y. Toda, "Bridgeland stability conditions on threefolds, I: Bogomolov-Gieseker type inequalities", preprint, 2011. To appear in J. Alg. Geom. arXiv 1103.5010v1

[Bridgeland 2007] T. Bridgeland, "Stability conditions on triangulated categories", Ann. of Math. (2) 166:2 (2007), 317-345. MR 2009c:14026 Zbl 1137.18008

[Bridgeland 2008] T. Bridgeland, "Stability conditions on K3 surfaces", Duke Math. J. 141:2 (2008), 241-291. MR 2009b:14030 Zbl 1138.14022 
[Bridgeland 2009] T. Bridgeland, "Spaces of stability conditions", pp. 1-21 in Algebraic geometry, Part 1 (Seattle, 2005), edited by D. Abramovich et al., Proc. Sympos. Pure Math. 80, Amer. Math. Soc., Providence, RI, 2009. MR 2010f:14011 Zbl 1169.14303

[Happel et al. 1996] D. Happel, I. Reiten, and S. O. Smalø, "Tilting in abelian categories and quasitilted algebras", Mem. Amer. Math. Soc. 120:575 (1996), 1-88. MR 97j:16009 Zbl 0849.16011

[Harris 1980] J. Harris, “The genus of space curves”, Math. Ann. 249:3 (1980), 191-204. MR 81i: 14022 Zbl 0449.14006

[Hartshorne 1977] R. Hartshorne, Algebraic geometry, Graduate Texts in Mathematics 52, Springer, New York, 1977. MR 57 \#3116 Zbl 0367.14001

[Hartshorne 1978] R. Hartshorne, "Stable vector bundles of rank 2 on $\mathbb{P}^{3}$ ", Math. Ann. 238:3 (1978), 229-280. MR 80c:14011 Zbl 0411.14002

[Huybrechts 2012] D. Huybrechts, "Introduction to stability conditions", preprint, 2012. arXiv 1111. $1745 \mathrm{v} 2$

[Lo and Qin 2011] J. Lo and Z. Qin, "Mini-walls for Bridgeland stability conditions on the derived category of sheaves over surfaces", preprint, 2011. arXiv 1103.4352v1

[Maciocia 2012] A. Maciocia, "Computing the walls associated to Bridgeland stability conditions on projective surfaces", preprint, 2012. To appear in Asian J. Math. arXiv 1202.4587v1

[Maciocia and Meachan 2013] A. Maciocia and C. Meachan, "Rank-1 Bridgeland stable moduli spaces on a principally polarized abelian surface", Int. Math. Res. Not. 2013:9 (2013), 2054-2077. MR 3053413

[Macrì 2007] E. Macrì, "Stability conditions on curves”, Math. Res. Lett. 14:4 (2007), 657-672. MR 2008k:18011 Zbl 1151.14015

[Minamide et al. 2011] H. Minamide, S. Yanagida, and K. Yoshioka, "Some moduli spaces of Bridgeland's stability conditions”, preprint, 2011. arXiv 1111.6187v3

[Polishchuk 2012] A. Polishchuk, "Phases of Lagrangian-invariant objects in the derived category of an abelian variety", preprint, 2012. arXiv 1203.2300v1

[Toda 2012a] Y. Toda, "Introduction and open problems of Donaldson-Thomas theory", pp. 289-318 in Derived categories in algebraic geometry (Tokyo, 2011), edited by Y. Kawamata, Eur. Math. Soc., Zürich, 2012. MR 3050708 Zbl 1256.14001

[Toda 2012b] Y. Toda, "Stability conditions and birational geometry of projective surfaces", preprint, 2012. To appear in Compos. Math. arXiv 1205.3602v1

[Toda 2013a] Y. Toda, "Bogomolov-Gieseker-type inequality and counting invariants", J. Topol. 6:1 (2013), 217-250. MR 3029426

[Toda 2013b] Y. Toda, "Stability conditions and extremal contractions", Math. Ann. 357:2 (2013), 631-685. MR 3096520 Zbl 06228499

[Yanagida and Yoshioka 2012] S. Yanagida and K. Yoshioka, "Bridgeland's stabilities on abelian surfaces", preprint, 2012. arXiv 1203.0884v1

[Yoshioka 2012] K. Yoshioka, "Bridgeland's stability and the positive cone of the moduli spaces of stable objects on an abelian surface", preprint, 2012. arXiv 1206.4838v1

Communicated by Yujiro Kawamata

Received 2012-07-30 Revised 2013-06-10 Accepted 2013-06-11

macri.6@math.osu.edu

Department of Mathematics, The Ohio State University, 231 West 18th Avenue, Columbus, OH 43210-1174, United States 


\section{Algebra \& Number Theory}

msp.org/ant

\section{EDITORS}

MANAGING EDITOR

Bjorn Poonen

Massachusetts Institute of Technology

Cambridge, USA

\author{
EDITORIAL BOARD CHAIR \\ David Eisenbud \\ University of California \\ Berkeley, USA
}

\section{BOARD OF EDITORS}

Georgia Benkart

Dave Benson

Richard E. Borcherds

John H. Coates

J-L. Colliot-Thélène

Brian D. Conrad

Hélène Esnault

Hubert Flenner

Edward Frenkel

Andrew Granville

Joseph Gubeladze

Roger Heath-Brown

Ehud Hrushovski

Craig Huneke

Mikhail Kapranov

Yujiro Kawamata

János Kollár

Yuri Manin

Barry Mazur

Philippe Michel
University of Wisconsin, Madison, USA

University of Aberdeen, Scotland

University of California, Berkeley, USA

University of Cambridge, UK

CNRS, Université Paris-Sud, France

University of Michigan, USA

Freie Universität Berlin, Germany

Ruhr-Universität, Germany

University of California, Berkeley, USA

Université de Montréal, Canada

San Francisco State University, USA

Oxford University, UK

Hebrew University, Israel

University of Virginia, USA

Yale University, USA

University of Tokyo, Japan

Princeton University, USA

Northwestern University, USA

Harvard University, USA

École Polytechnique Fédérale de Lausanne
Susan Montgomery

Shigefumi Mori

Raman Parimala

Jonathan Pila

Victor Reiner

Karl Rubin

Peter Sarnak

Joseph H. Silverman

Michael Singer

Vasudevan Srinivas

J. Toby Stafford

Bernd Sturmfels

Richard Taylor

Ravi Vakil

Michel van den Bergh

Marie-France Vignéras

Kei-Ichi Watanabe

Efim Zelmanov

Shou-Wu Zhang
University of Southern California, USA

RIMS, Kyoto University, Japan

Emory University, USA

University of Oxford, UK

University of Minnesota, USA

University of California, Irvine, USA

Princeton University, USA

Brown University, USA

North Carolina State University, USA

Tata Inst. of Fund. Research, India

University of Michigan, USA

University of California, Berkeley, USA

Harvard University, USA

Stanford University, USA

Hasselt University, Belgium

Université Paris VII, France

Nihon University, Japan

University of California, San Diego, USA

Princeton University, USA

PRODUCTION

production@msp.org

Silvio Levy, Scientific Editor

See inside back cover or msp.org/ant for submission instructions.

The subscription price for 2014 is US $\$ 225 /$ year for the electronic version, and $\$ 400 /$ year $(+\$ 55$, if shipping outside the US) for print and electronic. Subscriptions, requests for back issues and changes of subscribers address should be sent to MSP.

Algebra \& Number Theory (ISSN 1944-7833 electronic, 1937-0652 printed) at Mathematical Sciences Publishers, 798 Evans Hall \#3840, c/o University of California, Berkeley, CA 94720-3840 is published continuously online. Periodical rate postage paid at Berkeley, CA 94704, and additional mailing offices.

ANT peer review and production are managed by EditFLOW ${ }^{\circledR}$ from Mathematical Sciences Publishers.

\section{PUBLISHED BY}

- mathematical sciences publishers

nonprofit scientific publishing

http://msp.org/

(C) 2014 Mathematical Sciences Publishers 


\section{Algebra \& Number Theory}

\section{Volume $8 \quad$ No. $1 \quad 2014$}

On the Picard number of K3 surfaces over number fields

FRANÇOIS CHARLES

Adèle residue symbol and Tate's central extension for multiloop Lie algebras

OLIVER BRAUNLING

On the number of cubic orders of bounded discriminant having automorphism group $C_{3}$, and related problems

MANJUl Bhargava and ARIEl ShNidman

Polynomial bounds for Arakelov invariants of Belyi curves

ARIYAN JAVANPEYKAR

The radius of a subcategory of modules

HAILONG DAO and RYO TAKAHASHI

A generalized Bogomolov-Gieseker inequality for the three-dimensional projective space

EMANUELE MACRÌ

$(\varphi, \Gamma)$-modules over noncommutative overconvergent and Robba rings

GERGELY ZÁBRÁDI

The Tannakian formalism and the Langlands conjectures

DAVID KAZHDAN, MICHAEL LARSEN and YAKOV VARSHAVSKY 